\title{
SOME FEATURED OPTIMAL LAY-UP SOLUTIONS FOR COMPOSITE VAT PLATES UNDER BUCKLING, POSTBUCKLING AND LARGE-DEFLECTION POSTBUCKLING CONDITIONS
}

\author{
Sergey Selyugin \\ Stade, Germany \\ e-mail: sergey.selyugin@t-online.de
}

\begin{abstract}
The paper is devoted to some featured lay-up solutions for optimized composite VAT plates under such conditions as buckling, postbuckling (within the von Karman limits) or largedeflection postbuckling (beyond the von Karman limits). The corresponding optimality conditions are theoretically analyzed. The possible locally orthotropic solutions are identified and discussed. For the solutions the local orthotropy axes coincide with the principal (generalized) curvature axes.
\end{abstract}

Keywords: composite plate, lay-up, optimization, steered fibers, buckling, postbuckling, largedeflection postbuckling, local orthotropy

(C) Sergey Selyugin, 2021 


\section{Introduction}

Composite VAT (Variable Angle Tow or steered fibers) plates designed to withstand buckling or postbuckling loads are widely explored now numerically and in structural tests (see Turvey and Marshall, 1995, Falzon and Aliabadi, 2008, Reddy, 2007, Kassapoglu, 2010, Ghiasi, 2009, 2010).

It should be noted that the plates made of the so-called specially orthotropic materials and their plate-wise optimization were widely studied at the end of the previous century (see the numerous textbooks on structural optimization).

The theoretical first order necessary lay-up optimality conditions for the design cases indicated in the title of the present paper have been recently obtained (Selyugin 2019a, 2019b, 2021)

The present paper identifies and discusses some featured optimal lay-up solutions, namely, the locally orthotropic solutions. In our opinion, the solutions may be treated as the fundamental optimal lay-up solutions for the considered loadings.

\section{Theoretical consideration}

In the present paper we consider the laminated composite plate with the symmetric lay-up. The plate thickness is $h$. The lay-up is composed of the orthotropic fiber-reinforced plies of the same thickness $h_{p l y}$ and various point-wise orientation angles. The total number of plies is even and is equal to $2 \mathrm{~K}$, the generalization to the odd ply number is straightforward and is not considered in the present paper. Fig. 1 illustrates some notations used in the paper. 


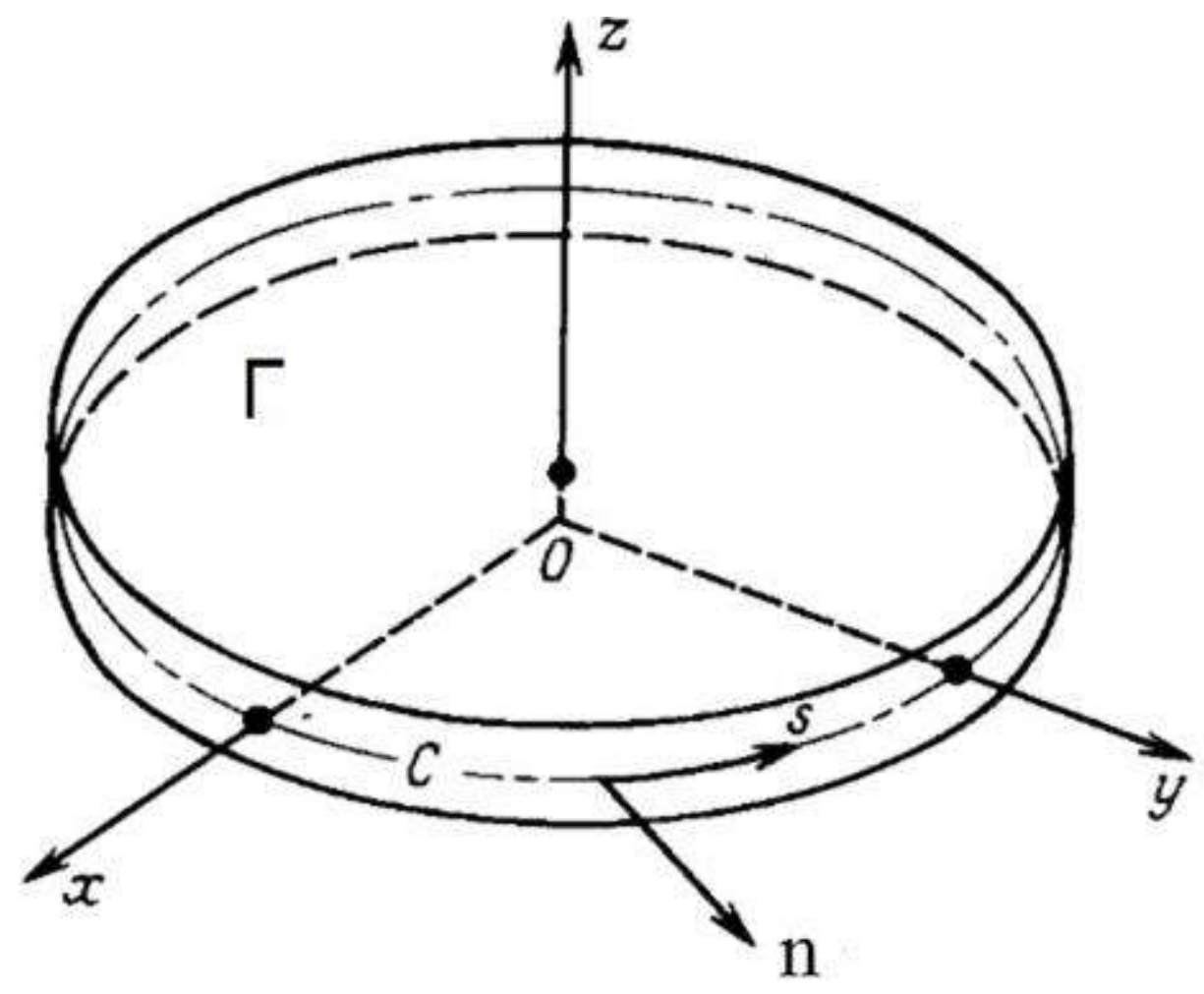

Fig. 1.

The mid-plane $\Gamma$ of the flat plate is located in $X Y$ plane. The mid-surface is restricted by the piecewise smooth contour $C$ with the external normal $n$ (the components of the normal vector are $l, m)$ and the tangent direction $s$; the directions $n, s$ and $\mathrm{Z}$ create a right-hand triplet. The coordinate system $X Y Z$ is a Cartesian one.

The plate is loaded by the in-plane forces, acting at the part $C_{1}$ of the contour $C$. The remaining part $C_{2}$ of the contour the plate is not moving in $X$ and $Y$.

The $X, Y, Z$ displacements are denoted as $u, v, w$, respectively.

The signs of the loads are the following. The force flows $N_{x}, N_{y}$ (along $X$ and along $Y$ ) are positive in tension, the shear force flow $N_{x y}$ is positive when it decreases the $90^{\circ}$ angle between the orthogonal lines at the plate surface. The flows are in equilibrium. The Classical Lamination Plate Theory (CLPT) is used for the description of the plate deflections (see the book of Gibson 1994). The components of the Green strain tensor are supposed to be small (negligible as compared to unity).

\subsection{Buckling-targeted lay-up solutions}

In this sub-Section the plate is supposed to be simply supported in $\mathrm{Z}$ direction.

As we have said, the Classical Lamination Plate Theory (CLPT), based on the linear-elastic relations for every layer and the Bernoulli's hypotheses, is used for obtaining the equation for the 
deflections $w$ (Gibson, 1994). The equilibrium equation in $z$ direction ( $x$ and $y$ correspond to Fig. $1)$ is written as follows:

$$
\begin{aligned}
& D_{11} \frac{\partial^{4} w}{\partial x^{4}}+2\left(D_{12}+2 D_{66}\right) \frac{\partial^{4} w}{\partial x^{2} \partial y^{2}}+D_{22} \frac{\partial^{4} w}{\partial y^{4}}+4 D_{16} \frac{\partial^{4} w}{\partial x^{3} \partial y}+4 D_{26} \frac{\partial^{4} w}{\partial x \partial y^{3}}- \\
& -N_{x} \frac{\partial^{2} w}{\partial x^{2}}-N_{y} \frac{\partial^{2} w}{\partial y^{2}}-2 N_{x y} \frac{\partial^{2} w}{\partial x \partial y}=0
\end{aligned}
$$

where $D_{i j}, i=1,2,6 ; j=1,2,6$, are the elements of the bending stiffness matrix $D$, coupling the bending/twisting moments and various second derivatives of the deflection $w$ with respect to $x$ and $y$. The coupling is written as follows:

$$
\left[\begin{array}{l}
M_{x} \\
M_{y} \\
M_{x y}
\end{array}\right]=\left[\begin{array}{lll}
D_{11} & D_{12} & D_{16} \\
D_{12} & D_{22} & D_{26} \\
D_{16} & D_{26} & D_{66}
\end{array}\right]\left[\begin{array}{c}
-\frac{\partial^{2} w}{\partial x^{2}} \\
-\frac{\partial^{2} w}{\partial y^{2}} \\
-2 \frac{\partial^{2} w}{\partial x \partial y}
\end{array}\right]
$$

We denote the left-hand side of (2) as the vector-column $\vec{M}$, and as $\vec{k}$ the vector-column at the right-hand side (which is multiplied to $D$ matrix). Also we use the notations

$$
k_{x}=-\frac{\partial^{2} w}{\partial x^{2}} ; k_{y}=-\frac{\partial^{2} w}{\partial y^{2}} ; k_{x y}=-\frac{\partial^{2} w}{\partial x \partial y}
$$

Then (2) is rewritten in the matrix-vector form as:

$$
\vec{M}=D \vec{k}
$$

According to (Selyugin 2019a) the lay-up optimality conditions in the problem are written as follows:

$$
\sin 2\left(\theta_{i}-\psi\right)\left[\frac{U_{2}}{4 U_{3}}\left(k_{1}^{2}-k_{2}^{2}\right)+\left(k_{1}-k_{2}\right)^{2} \cos 2\left(\theta_{i}-\psi\right)\right]=0, i=1, \ldots, K
$$

where $\theta_{i}, k_{1}, k_{2}, \psi, z_{i}, z_{i-1}$ respectively are the ply orientation angle, the largest and the lowest principal curvatures, the angle between the direction $k_{1}$ and the $\mathrm{X}$ axis, the top and the bottom $z$ coordinates of the $i$-th layer, $i=1, \ldots, K$.

The conditions (5) are identical with the ones obtained in (Selyugin, 2013) without an account of the changes of the in-plane forces due to the variation of the ply angles.

Analyzing the optimality conditions (5), it is possible to identify as one of the solutions the relation at every point

$$
\theta_{i}=\psi \text { or } \theta_{i}=\psi+\pi / 2, i=1, \ldots, K
$$

The solution is obviously a locally orthotropic lay-up and at every point the layers are oriented in the direction of the first or the second principal curvature.

In (Selyugin, 2013) it was shown that the linear combination of (5), regardless of the orientation of every layer, leads to a relation

$$
M_{x y}^{\text {pr.cur. }}\left(k_{1}-k_{2}\right)=0
$$


where $M_{x y}^{\text {pr.cur. }}$ is the twisting moment measured in the principal curvature axes. The relation (7) means that at every point the moment tensor and the tensor of curvatures are co-axial.

In (Selyugin, 2013) the deformation pattern (see Fig. 2) for a buckling-optimal shear-loaded long plate was shown. The pattern illustrates the possible orthotropic solution as well as the best material orientations.

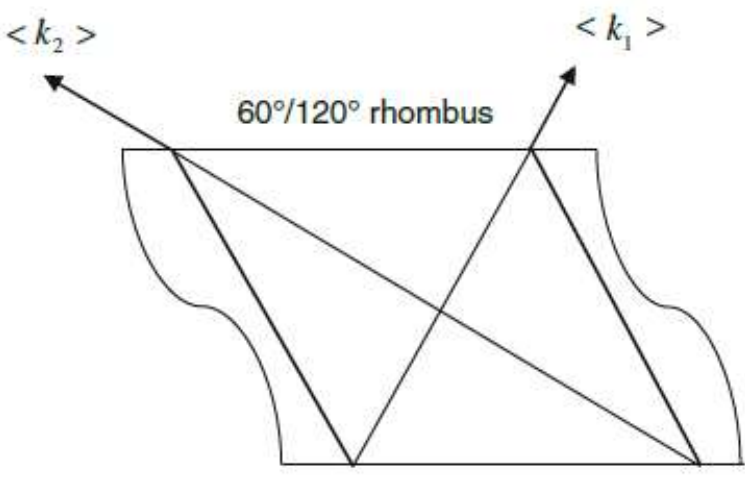

bay width

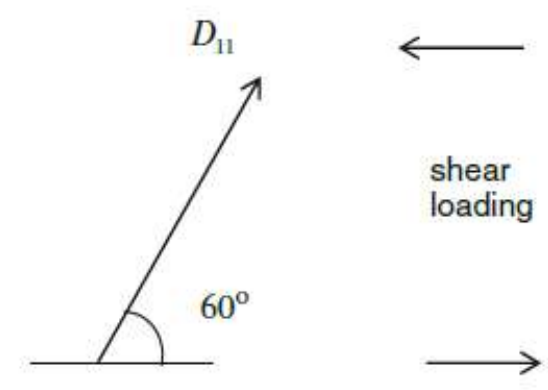

material direction 1

Fig. 2.

\subsection{Postbuckling-targeted lay-up solutions (von Karman limits)}

In (Selyugin 2019b) the lay-up optimality conditions for post-buckled VAT composite plates (in the von Karman limits) were obtained. The structural stiffness was optimized (the structural potential energy was considered as a measure of the stiffness).

Using two coordinate systems in the derivation, namely the first one with the axes along the principal 2D mid-plane strains $\varepsilon_{1}, \varepsilon_{2}$ directions (where $\varepsilon_{1} \geq \varepsilon_{2}$ ) and the second one with the axes along the principal plate curvatures $k_{1}, k_{2}$ (where $k_{1} \geq k_{2}$ ), we have for all $i=1, \ldots, K$ :

$$
\begin{aligned}
& \sin 2\left(\alpha_{i}-\varphi\right)\left[\frac{U_{2}}{4 U_{3}}\left(\varepsilon_{1}^{2}-\varepsilon_{2}^{2}\right)+\left(\varepsilon_{1}-\varepsilon_{2}\right)^{2} \cos 2\left(\alpha_{i}-\varphi\right)\right]+ \\
& +\sin 2\left(\alpha_{i}-\xi\right)\left[\frac{U_{2}}{4 U_{3}}\left(k_{1}^{2}-k_{2}^{2}\right)+\left(k_{1}-k_{2}\right)^{2} \cos 2\left(\alpha_{i}-\xi\right)\right] \frac{1}{3}\left(z_{i}^{2}+z_{i} z_{i-1}+z_{i-1}^{2}\right)=0
\end{aligned}
$$

where $\varphi$ is the angle between the global $x$-axis and the $\varepsilon_{1}$ axis; $\xi$ is the angle between the global $x$-axis and the $k_{1}$ axis; $U_{2}, U_{3}$ are some elastic constants of the ply (see Gibson, 1994, $\S 2.6), z_{i}$ and $z_{i-1}$ respectively are the top and the bottom $z$ coordinates of the $i$-th ply.

The relations (8) are, in particular, valid when both sines are equal to zero. This means that

$$
\begin{gathered}
\varphi=\xi \text { or } \varphi=\xi+\pi / 2 \text { and } \\
\alpha_{i}=\varphi \text { or } \alpha_{i}=\xi \text { (for every } i \text { ) }
\end{gathered}
$$

are the solutions of (8). The solutions correspond to the locally orthotropic laminate.

It was shown in (Selyugin, 2019b) that, summing the conditions (8), we obtain 


$$
N_{x y}^{p r . s t r .}\left(\varepsilon_{1}-\varepsilon_{2}\right)+M_{x y}^{p r . c u r .}\left(k_{1}-k_{2}\right)=0
$$

where pr.str., pr.cur. correspond to the principal mid-plane strain axes and to the principal curvature axes.

The relation (10) is a linear combination of the optimality conditions (8) and, hence, also is the optimality condition uniting the ones for every layer. The first item in (10), if considered separately as equal to zero, means that the nonlinear mid-plane strain tensor is co-axial with the force tensor (or, for $\varepsilon_{1} \neq \varepsilon_{2}$, the shear flow in the principal strain axes is equal to zero). The second item in (10), if considered separately as equal to zero, means that the tensor of the curvatures is co-axial with the moment tensor (or, for $k_{1} \neq k_{2}$, the twisting moment in the principal curvature axes is equal to zero).

When the conditions (9) are valid, both $N_{x y}^{\text {pr.str. }}$ and $M_{x y}^{\text {pr.cur. }}$ are equal to zero. This means that the principal strain axes coincide with the principal curvature axes. These orthogonal directions are the directions of the local orthotropy.

\subsection{Large-deflection postbuckling-targeted lay-up solutions (beyond the von Karman limits)}

Small elongations, small shears and small squared in-plane rotations (negligible as compared to unity) are assumed. Following (Novozhilov, 1961, 2011), the displacements $u, v, w$ within the plate (respectively along $\mathrm{X}, \mathrm{Y}, \mathrm{Z}$ ) are supposed to be

$$
\begin{aligned}
& u(x, y)=\hat{u}(x, y)+z \cdot \theta(x, y) \\
& \mathrm{v}(x, y)=\hat{\mathrm{v}}(x, y)+z \cdot \psi(x, y) \\
& w(x, y)=\hat{w}(x, y)+z \cdot \chi(x, y)
\end{aligned}
$$

where upper 'tilde' means quantities at the mid-plane.

After using (11) and the definition of the Green tensor components it is obtained in (Novozhilov, 1961, 2011) that

$$
\begin{aligned}
& \theta=-\hat{w},_{x}\left(1+\hat{\mathrm{v}},{ }_{y}\right)+\hat{\mathrm{v}},{ }_{x} \hat{w},{ }_{y} \\
& \psi=-\hat{w},{ }_{y}\left(1+\hat{u},{ }_{x}\right)+\hat{u},{ }_{y} \hat{w}_{x} \\
& \chi=\hat{u},{ }_{x}+\hat{\mathrm{v}},{ }_{y}+\hat{u},{ }_{x} \hat{\mathrm{v}},{ }_{y}-\hat{u},{ }_{y} \hat{\mathrm{v}},{ }_{x}
\end{aligned}
$$

and

$$
\begin{aligned}
& \varepsilon_{x x}=\hat{\varepsilon}_{x x}+z \kappa_{x x}+z^{2} \eta_{x x} \\
& \varepsilon_{y y}=\hat{\varepsilon}_{y y}+z \kappa_{y y}+z^{2} \eta_{y y} \\
& \varepsilon_{x y}=\hat{\varepsilon}_{x y}+z \kappa_{x y}+z^{2} \eta_{x y}
\end{aligned}
$$

The mid-plane strains in (13) are 


$$
\begin{aligned}
& \hat{\varepsilon}_{x x}=\hat{u},_{x}+1 / 2\left[\hat{u},{ }_{x}^{2}+\hat{\mathrm{v}},{ }_{x}^{2}+\hat{w},{ }_{x}^{2}\right] \\
& \hat{\varepsilon}_{y y}=\hat{\mathrm{v}},_{y}+1 / 2\left[\hat{u},{ }_{y}^{2}+\hat{\mathrm{v}},{ }_{y}^{2}+\hat{w},{ }_{y}^{2}\right] \\
& 2 \hat{\varepsilon}_{x y}=\hat{u},_{y}+\hat{\mathrm{v}},_{x}+\hat{u},{ }_{y} \hat{u},{ }_{x}+\hat{\mathrm{v}},{ }_{x} \hat{\mathrm{v}},_{y}+\hat{w},{ }_{x} \hat{w},_{y}
\end{aligned}
$$

and other quantities in (13):

$$
\begin{aligned}
& \kappa_{x x}=\theta,_{x}+\hat{u},{ }_{x} \theta,{ }_{x}+\hat{\mathrm{v}},{ }_{x} \psi,,_{x}+\hat{w},{ }_{x} \chi,_{x} \\
& \kappa_{y y}=\psi,{ }_{y}+\hat{u},{ }_{y} \theta,{ }_{y}+\hat{\mathrm{v}},{ }_{y} \psi,{ }_{y}+\hat{w},{ }_{y} \chi,_{y} \\
& 2 \kappa_{x y}=\theta,_{y}+\psi,_{x}+\hat{u},_{x} \theta,_{y}+\hat{u},_{y} \theta,_{x}+\hat{\mathrm{v}},_{x} \psi,,_{y}+\hat{\mathrm{v}},{ }_{y} \psi,_{x}+\hat{w},,_{x} \chi,_{y}+\hat{w},{ }_{y} \chi,_{x} \\
& \eta_{x x}=1 / 2\left[\theta,{ }_{x}^{2}+\psi,{ }_{x}^{2}+\chi,_{x}^{2}\right] \\
& \eta_{y y}=1 / 2\left[\theta,{ }_{y}^{2}+\psi,{ }_{y}^{2}+\chi,{ }_{y}^{2}\right] \\
& 2 \eta_{x y}=\theta,_{x} \theta,,_{y}+\psi,{ }_{x} \psi,{ }_{y}+\chi,{ }_{x} \chi,{ }_{y}
\end{aligned}
$$

Hereinafter we name (15) the generalized curvatures. In (Novozhilov, 1961, 2011) it is indicated that for small elongations and small shears the last terms in every relation of (13) are small enough and may be neglected. Then (13) is transformed into

$$
\begin{aligned}
& \varepsilon_{x x}=\hat{\varepsilon}_{x x}+z \kappa_{x x} \\
& \varepsilon_{y y}=\hat{\varepsilon}_{y y}+z \kappa_{y y} \\
& \varepsilon_{x y}=\hat{\varepsilon}_{x y}+z \kappa_{x y}
\end{aligned}
$$

Substituting (12) in (15) and keeping in mind the small in-plane rotations, after cumbersome transformations we obtain (see Selyugin 2021)

$$
|\chi|<<1
$$

and the generalized curvatures

$$
\begin{aligned}
& \kappa_{x x}=-\theta(x, y) \hat{u},_{x x}-\psi(x, y) \hat{\mathrm{v}},_{x x}-\hat{w},_{x x} \\
& \kappa_{y y}=-\theta(x, y) \hat{u},_{y y}-\psi(x, y) \hat{\mathrm{v}},_{y y}-\hat{w},_{y y} \\
& \kappa_{x y}=-\theta(x, y) \hat{u},_{x y}-\psi(x, y) \hat{\mathrm{v}},_{x y}-\hat{w},_{x y}
\end{aligned}
$$

In (Selyugin, 2021) the plate stiffness optimization problem in the above conditions was studied (the structural potential energy was considered a measure of the stiffness). The plate layup was varied. The lay-up optimality conditions were obtained, $i=1, \ldots, K$.

Using two coordinate systems in the derivation, namely, the first one with the axes along the principal 2D mid-plane strains $\varepsilon_{1}, \varepsilon_{2}$ directions (where $\varepsilon_{1} \geq \varepsilon_{2}$ ) and the second one with the axes along the principal plate generalized curvatures $k_{1}, k_{2}$ (where $k_{1} \geq k_{2}$ ), we have for all $i=1, \ldots, K$ : 


$$
\begin{aligned}
& \sin 2\left(\alpha_{i}-\varphi\right)\left[\frac{U_{2}}{4 U_{3}}\left(\varepsilon_{1}^{2}-\varepsilon_{2}^{2}\right)+\left(\varepsilon_{1}-\varepsilon_{2}\right)^{2} \cos 2\left(\alpha_{i}-\varphi\right)\right]+ \\
& +\sin 2\left(\alpha_{i}-\xi\right)\left[\frac{U_{2}}{4 U_{3}}\left(k_{1}^{2}-k_{2}^{2}\right)+\left(k_{1}-k_{2}\right)^{2} \cos 2\left(\alpha_{i}-\xi\right)\right] \frac{1}{3}\left(z_{i}^{2}+z_{i} z_{i-1}+z_{i-1}^{2}\right)=0
\end{aligned}
$$

where $\varphi$ is the angle between the global $x$-axis and the $\varepsilon_{1}$ axis; $\xi$ is the angle between the global $x$-axis and the $k_{1}$ axis; $U_{2}, U_{3}$ are some elastic constants of the ply (see Gibson, 1994, $\S 2.6), z_{i}$ and $z_{i-1}$ respectively are the top and the bottom $z$ coordinate of the $i$-th ply.

The relations (20) are, in particular, valid when both sines are equal to zero. This means that

$$
\begin{gathered}
\varphi=\xi \text { or } \varphi=\xi+\pi / 2 \text { and } \\
\alpha_{i}=\varphi \text { or } \alpha_{i}=\xi \text { (for every } i \text { ) }
\end{gathered}
$$

are the solutions of (20). The solutions correspond to the locally orthotropic laminate.

It was shown in (Selyugin, 2021) that, summing the conditions (20), we obtain

$$
N_{x y}^{\text {pr.str. }}\left(\varepsilon_{1}-\varepsilon_{2}\right)+M_{x y}^{\text {pr.cur. }}\left(k_{1}-k_{2}\right)=0
$$

where pr.str., pr.cur. correspond to the principal mid-plane strain axes and to the principal generalized curvature axes.

The relation (22) is a linear combination of the optimality conditions (20), and, hence, also is the optimality condition uniting the ones for every layer. The first item in (22), if considered separately as equal to zero, means that the nonlinear mid-plane strain tensor is co-axial with the force tensor (or, for $\varepsilon_{1} \neq \varepsilon_{2}$, the shear flow in the principal strain axes is equal to zero). The second item in (22), if considered separately as equal to zero, means that the tensor of the generalized curvatures is co-axial with the moment tensor (or, for $k_{1} \neq k_{2}$, the twisting moment in the principal generalized curvature axes is equal to zero).

When the conditions (21) are valid, both $N_{x y}^{p r . s t r}$ and $M_{x y}^{p r . c u r .}$ are equal to zero. This means that the principal strain axes coincide with the principal generalized curvature axes. These orthogonal directions are the directions of the local orthotropy.

\section{Discussion}

In addition to the above-discussed cases, it is known (see, e.g., Pedersen 1989) that some locally orthotropic solutions are valid for the plate 2D stiffness optimization in statics. It is also obvious, in view of the above results that the plate stiffness optimization in bending-twistingtension leads to the locally orthotropic solutions.

\section{Conclusions}


- Some optimal lay-up solutions for the considered optimization cases are the locally orthotropic ones. For the solutions the local orthotropy axes coincide with the principal (generalized) curvature axes;

- The locally orthotropic lay-up may be treated as a fundamental one in the considered optimization cases;

- The locally orthotropic lay-up may be recommended as a design approach for the practical structures.

\section{REFERENCES}

Falzon BG, Aliabadi MH 2008. Buckling and postbuckling structures: experimental, analytical and numerical studies, Imperial College Press.

Ghiasi H, Pasini D, Lessard L 2009. Optimum Stacking Sequence Design of Composite Materials Part I: Constant Stiffness Design. Compos Struct 90: 1-11.

Ghiasi H, Fayazbakhsh K, Pasini D, Lessard L 2010. Optimum Stacking Sequence Design of Composite Materials Part II: Variable Stiffness Design. Compos Struct 93: 1-13

Gibson RF 1994. Principles of composite material mechanics. McGraw-Hill, Inc., 425 pp.

Kassapoglou C 2010. Design and Analysis of Composite Structures: With Applications to Aerospace Structures. Wiley.

Novozhilov V, 2011. Foundations of the nonlinear theory of elasticity. Dover Publ., 256 pp.

Novozhilov V, 1961. Theory of elasticity. Pergamon Press, 448 pp.

Pedersen P, 1989. On optimal orientation of orthotropic materials. Structural Optimization, 1, 101-106.

Reddy JN, 2007. Theory and analysis of elastic plates and shells. $2^{\text {nd }}$ edition. Taylor $\&$ Francis Group, New York.

Selyugin S 2013. On Choice of Optimal Anisotropy of Composite Plates against Buckling, with Special Attention to Bending-twisting Coupling. Struct Multidisc Optim 48: 279-294

Selyugin S 2019a. Lay-up optimality conditions for buckling level maximization of VAT (steered fiber) composite plates. https://doi.org/10.31224/osf.io/g7v2a 
Selyugin S 2019b. Lay-up optimality conditions for stiffness maximization of anisotropic composite plates in postbuckling. https://doi.org/10.31224/osf.io/k2rwp

Selyugin S 2021. Locally orthotropic lay-up as an optimal solution for VAT post-buckled composite plates experiencing large deflections above von Karman limits. https://doi.org/10.31224/osf.io/4a6hz

Turvey GJ, Marshall IH (eds) 1995. Buckling and postbuckling of composite plates. Chapman \& Hall. 